\title{
Avaliação de vida residual de tubos de forno reformador
}

\author{
Remaining life evaluation of reformer \\ furnace catalyst tubes
}

Bagagli, R. P.; Ventorin, A.; Secolin, H.

Inspeção de Equipamentos PETROBRAS/REPLAN, Rod. SP 332 Km 130, Paulínia, SP. e-mail: rafaelpavan@petrobras.com.br ; aislan.ventorin@ petrobras.com.br ; secolin@ petrobras.com.br

\section{RESUMO}

Este trabalho avalia a vida remanescente dos tubos catalisadores de um forno reformador devido ao fato do fim da vida de projeto de 100.000 horas estar próximo.

O principal mecanismo de dano destes tubos é a fluência, por isso a análise seguiu roteiro da Part 10 do documento API-579/ASME FFS-1 (2007).

O material dos tubos é uma liga HP modificada, para a qual existe escassez de dados de propriedades na literatura. Por isso, as propriedades de fluência necessárias para a avaliação foram determinadas experimentalmente utilizando correlação de dados de ensaios de tração a quente e fluência.

Os resultados indicam que os tubos não necessitam ser substituídos de imediato e que sua vida residual é estimada em 22,3 anos operando na temperatura de projeto

Palavras-chave: Forno reformador, HP modificado, fluência.

\section{ABSTRACT}

This work evaluates remaining life of catalyst tubes of a reformer furnace due to achieving end of design life (100.000 hours).

The main damage mechanism in this case is creep, hence the analysis was based in the procedure of API579/ASME FFS-1 - Part 10 (2007).

The tubes material is the alloy HP modified, which there isn't enough creep properties data on literature. Therefore, these properties have been defined based on experimental data from hot tensile and creep tests.

The results showed that is not necessary to replace the tubes immediately and the remaining life is about 22 years operating at design temperature.

Keywords: Reformer furnace, modified HP, creep.

\section{INTRODUÇÃO}

O principal mecanismo de dano dos tubos catalisadores da seção de radiação de fornos reformadores de unidades geradoras de hidrogênio (UGH’s) é a fluência. Por isso, são projetados conforme a norma API-530 para uma vida de 100.000h (aproximadamente 11 anos) em determinada temperatura e pressão de projeto.

Devido o fim de vida de projeto dos tubos de um dos fornos reformadores da Refinaria de Paulínia (REPLAN) da Petrobras estar próximo, foi necessário realizar uma avaliação de integridade e estimativa de vida residual em alguns níveis de temperatura de operação para verificar se era possível prolongar a substituição após a parada de 2009. 
O material dos tubos é uma liga do tipo 25Cr-35Ni+Nb (HP modificado) fundido por centrifugação e bastante resistente à fluência em altas temperaturas. Porém, não existem dados consolidados na literatura sobre as propriedades deste material para realização de uma avaliação de vida residual. Por isso, foram retirados dois tubos na parada para confecção de corpos de prova e obtenção experimental das propriedades necessárias para a estimativa de vida residual.

Depois de obtidas as propriedades necessárias, a avaliação foi realizada utilizando procedimentos do documento API-579/ASME FFS-1 Part 10 [1].

\section{RESULTADOS E DISCUSSÕES}

Para a avaliação de fluência segundo a Part 10 do API-579/ASME FFS-1, é necessário o levantamento do histórico de operação para definição dos dados de tempo x temperatura $\mathrm{x}$ tensão para cálculo do dano por fluência.

No entanto, o referido documento permite utilizar dados obtidos em ensaios de amostras do material e estimar a vida residual do material a partir da condição atual, ou seja, com o dano passado já embutido na análise.

Existem duas metodologias para avaliação de fluência pelo API-579/ASME FFS-1 Part 10: utilização do "parâmetro Omega” ou utilização do "parâmetro de Larson-Miller”. Como não existem dados suficientes na literatura sobre aplicabilidade de ambos os métodos para o material HP modificado, foram sacados dois tubos na parada de fevereiro de 2009 para retirada de amostras e realização de ensaios para avaliar qual dos dois é mais adequado para este material.

\subsection{Definição das propriedades do material}

Para definir as propriedades do material necessárias à avaliação da vida residual, foram realizados ensaios de fluência no laboratório da STM - Sistemas de Testes em Materiais - São Carlos/SP conforme características abaixo:

- Tipo: Isotensão (tensão fixa, 03 condições de temperatura);

- Tensão: 6,5 MPa (tensão equivalente de Von-Mises);

- Temperaturas: $790^{\circ} \mathrm{C} / 890^{\circ} \mathrm{C} / 980^{\circ} \mathrm{C}$, alterado para $1.050^{\circ} \mathrm{C} / 1.100^{\circ} \mathrm{C} / 1.150^{\circ} \mathrm{C}$ após algumas horas de ensaio;

- Tempo máximo: 1.000 horas/ensaio mesmo que não tenha ocorrido rompimento do corpo de prova;

- Amostras: 06 retiradas no sentido longitudinal (03 de cada tubo).

As curvas deformação x tempo obtidas nos ensaios de fluência são mostradas nas Figuras 1 a 3.

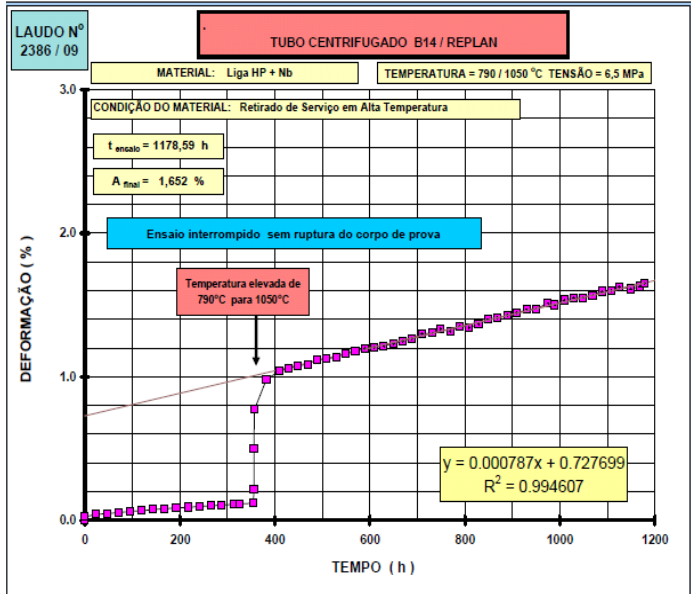

(a)

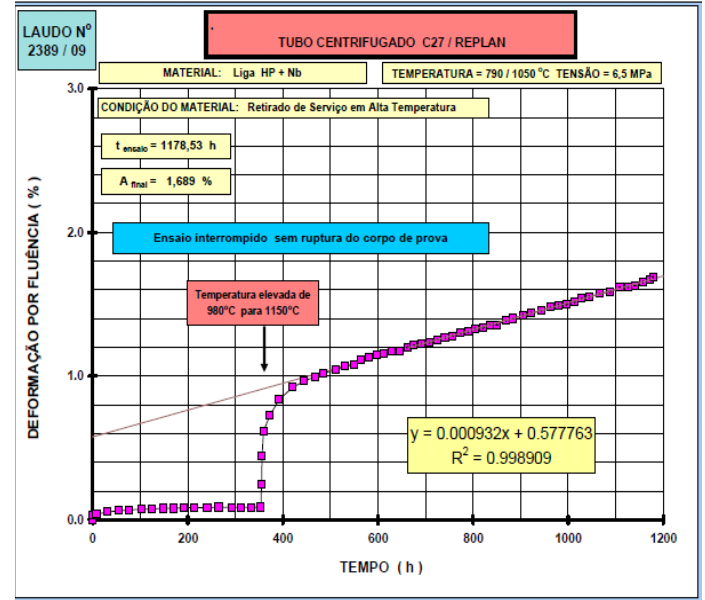

(b)

Figura 1: Curva deformação x tempo para a temperatura de $1.050^{\circ} \mathrm{C}$. Não houve rompimento do corpo de prova. 


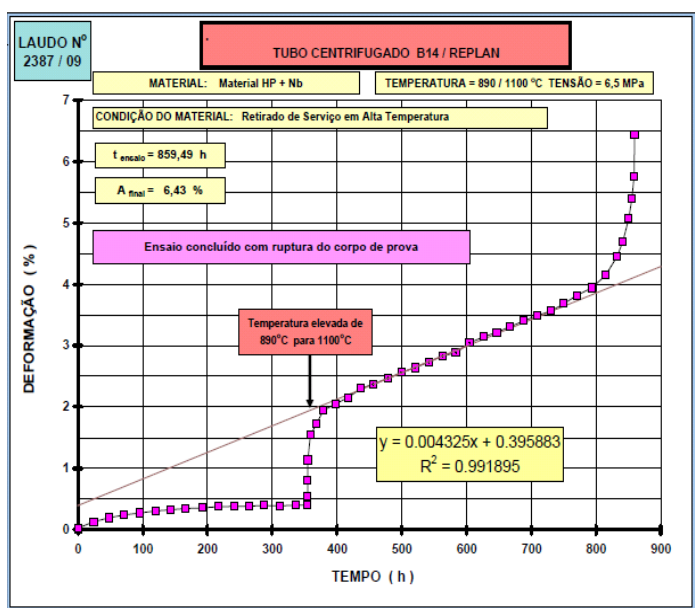

(a)

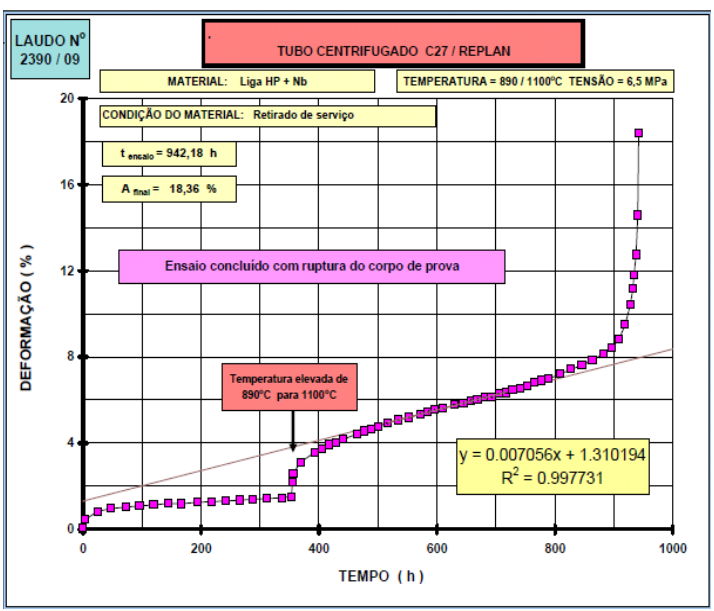

(b)

Figura 2: Curva deformação x tempo para a temperatura de $1.100^{\circ} \mathrm{C}$. Ruptura do corpo de prova para (a) tubo 01 em 859,49 horas e (b) tubo 02 após 942,18 horas.

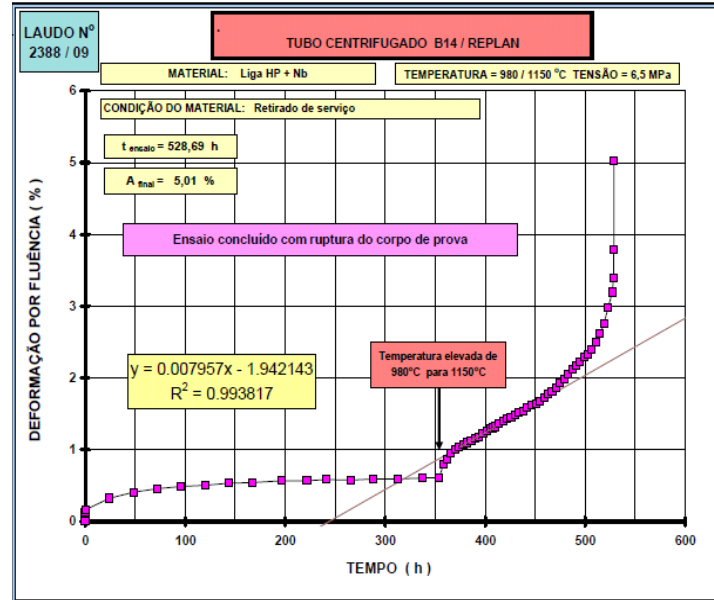

(a)

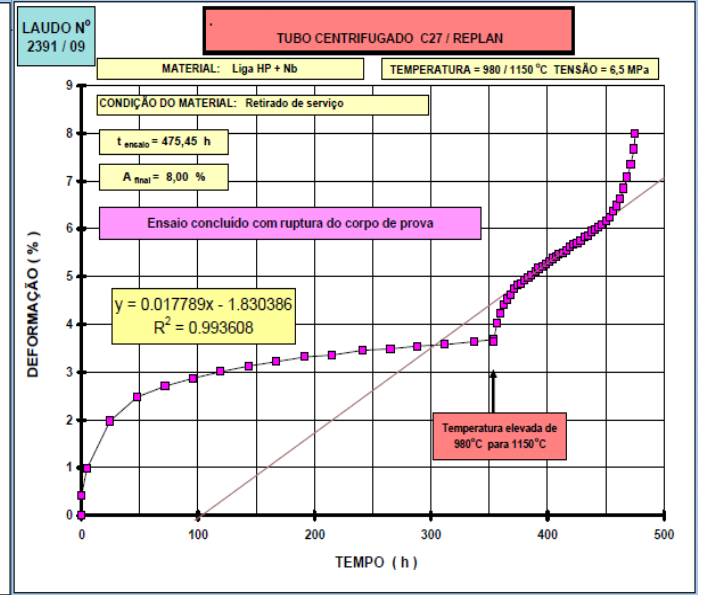

(b)

Figura 3: Curva deformação x tempo para a temperatura de $1.150^{\circ} \mathrm{C}$. Ruptura do corpo de prova para (a) tubo 01 em 528,69 horas e (b) tubo 02 após 475,45 horas. abaixo:

Além dos ensaios de fluência, foram realizados 09 ensaios de tração a quente conforme as condições

- $890^{\circ} \mathrm{C}$ com velocidades de 20,0 - 0,4 - 0,1 mm/min;

- $1.050^{\circ} \mathrm{C}$ com velocidades de $20,0-0,4 \mathrm{~mm} / \mathrm{min}$;

- $1.100^{\circ} \mathrm{C}$ com velocidade de $20,0-0,4-0,1 \mathrm{~mm} / \mathrm{min}$;

- $1.150^{\circ} \mathrm{C}$ com velocidade de $0,1 \mathrm{~mm} / \mathrm{min}$.

Os resultados dos ensaios de tração a quente estão resumidos na Tabela 2 do item 2.1.2 e foram utilizados em conjunto com os ensaios de fluência para determinar a curva de Larson-Miller conforme metodologia proposta por [2]. 


\subsubsection{Definição de omega $(\Omega)$ e da taxa de deformação inicial $\left(\varepsilon_{\text {co }}\right)$}

Na metodologia Omega devem ser definidos dois parâmetros a partir dos dados de deformação x tempo oḅtidos no ensaio de fluência para cálculo do tempo de ruptura: o parâmetro $\Omega$ e a taxa inicial de deformação $\varepsilon_{c o}$.

Segundo a referência [3], o parâmetro $\Omega$ é definido como sendo a variação da taxa de deformação em relação à própria deformação conforme:

$$
\Omega=d(\ln \dot{\varepsilon}) / d \varepsilon
$$

Portanto, para definição dos parâmetros acima, bastạ traçar a curva $\ln (\dot{\varepsilon})$ x $\varepsilon$ e tirar o coeficiente de inclinação de reta $(\Omega)$ e o ponto de intersecção no eixo y $\left(\varepsilon_{c o}\right)$.

Uma vez definidos os parâmetros $\Omega$ e $\dot{\varepsilon_{c o}}$, é possível definir o tempo para ruptura $(L)$ para cada condição de temperatura de ensaio utilizando a equação:

$$
L=\frac{1}{\varepsilon_{\mathrm{CO}} \Omega}
$$

A Tabela 1 mostra os parâmetros calculados para cada ensaio e a comparação do tempo de ruptura obtido da equação com o tempo de ruptura real do corpo de prova.

\begin{tabular}{|c|c|c|c|c|c|c|}
\hline AMOSTRA & $\mathrm{T}\left[{ }^{\circ} \mathrm{C}\right]$ & $\Omega$ & $\varepsilon_{\text {co }}^{\circ} \% /$ HORA $]$ & L [HORAS] & $t_{\text {rup }} B$ [HORAS] & $\begin{array}{l}\text { DIFERENÇA } \\
\left(L-t_{\text {rup }}\right)\end{array}$ \\
\hline \multirow[t]{3}{*}{ C-27 } & $1050^{*}$ & - & - & - & - & - \\
\hline & 1100 & 172,637 & 2,37e-07 & 24420 & 496 & 24246 \\
\hline & 1150 & 104,209 & 4,23e-05 & 227 & 175 & 52 \\
\hline \multirow[t]{3}{*}{ B-14 } & $1050^{*}$ & - & - & - & - & - \\
\hline & 1100 & 66,874 & 4,19e-06 & 3573 & 589 & 2984 \\
\hline & 1150 & 117,708 & 2,2e-05 & 380 & 122 & 285 \\
\hline
\end{tabular}

Tabela 1: Valores de Omega e taxa de deformação inicial, obtidos a partir das curvas de fluência.

* Não atingiu estágio terciário em 1000 horas

É possível notar na Tabela 1 que os tempos previstos pela metodologia Omega $(L)$ foram significativamente diferentes dos tempos de ruptura dos ensaios $\left(t_{\text {rup }}\right)$. Esta diferença invalida a utilização desta metodologia para extrapolação de dados e estimativa de vida residual.

Na verdade, a metodologia Omega modela o terceiro estágio de fluência supondo pouca significância do primeiro e segundo estágio. No entanto, as curvas de fluência obtidas nos ensaios mostram que há uma parcela significativa do segundo estágio (trecho linear das curvas), o que provoca uma incerteza nos resultados obtidos pela projeção Omega.

Segundo a referência [3], é possível verificar se a metodologia Omega é válida para um determinado conjunto de dados obtidos em ensaio de fluência se a curva da deformação $(\varepsilon)$ em função da diferença logarítmica entre o tempo instantâneo e o tempo de ruptura (log[t- $\left.\left.t_{\text {ruptura }}\right]\right)$ se aproximar de uma reta. A Figura 4 mostra estas curvas para os ensaios realizados. 

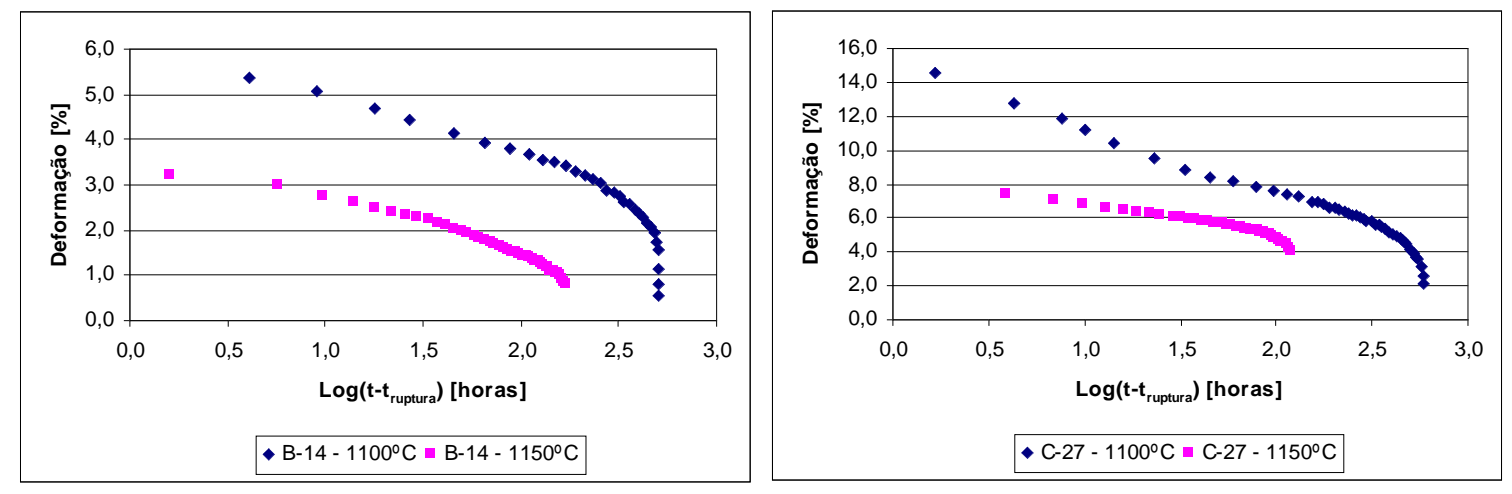

Figura 4: Gráficos da deformação x $\log \left(\mathrm{t}-\mathrm{t}_{\text {ruptura }}\right)$. a) Amostras tubo 1 e (b) Amostras tubo 2

A Figura 4 mostra que a curva deformação $\mathrm{x} \log \left(\mathrm{t}-\mathrm{t}_{\text {ruptura }}\right)$ possui um decaimento pronunciado, o que afasta seu comportamento ao de uma reta. Isto evidencia a influência do segundo estágio nestes ensaios e invalida a utilização do método Omega para estimativa de vida residual.

\subsubsection{Definição da curva mestre do parâmetro de Larson-Miller}

A definição do parâmetro de Larson-Miller foi baseada na metodologia proposta em [2], onde os autores demonstraram ser possível, para um aço ferrítico 2,25Cr-1,0Mo, correlacionar os resultados de ensaios de tração a quente com resultados de ensaios de fluência para obtenção da curva mestre da tensão em função do referido parâmetro.

O material da referência citada não é o mesmo deste trabalho, no entanto foi utilizada a mesma idéia de correlação de dados de ensaios de tração a quente e fluência. Os critérios para correlação são:

1.A taxa de deformação em um ensaio de tração é equivalente à taxa mínima de deformação obtida em um ensaio de fluência.

2.A tensão de ruptura obtida no ensaio de tração (início da estricção) é equivalente à tensão aplicada no ensaio de fluência.

3.O tempo para ocorrer a tensão de ruptura do material no ensaio de tração (início da estricção) é equivalente ao tempo de ruptura obtido no ensaio de fluência.

A partir da conversão dos dados de tração a quente em fluência é possível definir o parâmetro de Larson-Miller (PLM) conforme equação:

$$
P L M=[T+273]\left[C+\log \left(t_{R}\right)\right]
$$

$T$ - temperatura do ensaio em ${ }^{\circ} \mathrm{C}$;

$t_{R}$ - tempo de ruptura em horas;

$C$ - constante que depende do material.

Uma questão importante é o valor da constante $C$ a ser utilizada na definição do parâmetro de LarsonMiller, pois sua obtenção experimental é bastante trabalhosa, uma vez que depende de ensaios em muitas condições de tensão e temperatura para obtenção do gráfico $\sigma$ x 1/T. O valor de $C$ utilizado neste trabalho foi de 22,96, obtido da folha de dados fabricante do forno.

A Tabela 2 mostra os dados de tração a quente e fluência obtidos nos ensaios e utilizados na construção das curvas de Larson-Miller: 
Tabela 2: Dados de tração à quente e fluência utilizados para construção da curva PLM x $\sigma$.

\begin{tabular}{|c|c|c|c|c|c|c|}
\hline TUBO & ENSAIO & $\begin{array}{c}\mathbf{T} \\
{\left[{ }^{\circ} \mathrm{C}\right]}\end{array}$ & $\begin{array}{c}\sigma \\
{[\mathrm{MPa}]}\end{array}$ & $\begin{array}{c}\log (\sigma) \\
{[\mathrm{MPa}]}\end{array}$ & $\mathbf{t}_{\text {rup }}$ & PLM \\
\hline \multirow{11}{*}{ B-14 } & \multirow{9}{*}{$\begin{array}{l}\text { Tração a } \\
\text { Quente }\end{array}$} & 890 & 236,1 & 2,37 & 0,003 & 20304,2 \\
\hline & & 890 & 127,7 & 2,11 & 0,141 & 22270,9 \\
\hline & & 890 & 66,1 & 1,82 & 4,014 & 23961,9 \\
\hline & & 1050 & 104,6 & 2,02 & 0,003 & 23081,2 \\
\hline & & 1050 & 49,8 & 1,70 & 0,129 & 25285,3 \\
\hline & & 1100 & 80,2 & 1,90 & 0,002 & 23692,8 \\
\hline & & 1100 & 40,8 & 1,61 & 0,088 & 26013,0 \\
\hline & & 1100 & 22,9 & 1,36 & 1,847 & 27825,9 \\
\hline & & 1150 & 29,7 & 1,47 & 0,136 & 27225,0 \\
\hline & \multirow{2}{*}{ Fluência } & 1100 & 6,5 & 0,81 & 495,86 & 31160,7 \\
\hline & & 1150 & 6,5 & 0,81 & 174,54 & 31650,2 \\
\hline \multirow{10}{*}{ B-14 } & \multirow{8}{*}{$\begin{array}{l}\text { Tração a } \\
\text { Quente }\end{array}$} & 890 & 236,7 & 2,37 & 0,003 & 20266,1 \\
\hline & & 890 & 120,9 & 2,08 & 0,115 & 22167,6 \\
\hline & & 890 & 64,4 & 1,81 & 3,486 & 23890,7 \\
\hline & & 1050 & 102,1 & 2,01 & 0,003 & 23081,2 \\
\hline & & 1050 & 51,4 & 1,71 & 0,077 & 24984,3 \\
\hline & & 1100 & 78,4 & 1,89 & 0,002 & 23603,9 \\
\hline & & 1100 & 37,6 & 1,58 & 0,069 & 25869,6 \\
\hline & & 1100 & 20,9 & 1,32 & 2,528 & 28013,0 \\
\hline & \multirow{2}{*}{ Fluência } & 1100 & 6,5 & 0,81 & 589,18 & 31263,6 \\
\hline & & 1150 & 6,5 & 0,81 & 121,65 & 31427,1 \\
\hline
\end{tabular}

Definido o PLM para cada ensaio, a curva mestre PLM x Tensão é construída. Com essa relação é possível extrapolar a avaliação para qualquer condição de temperatura e tensão. É importante ressaltar que devem ser utilizados alguns dados de ensaios de fluência para verificar se realmente existe a tendência de correlação.

A metodologia pode ser validada construindo algumas curvas de relações clássicas de fluência compostas com dados de ambos os ensaios e verificar o alinhamento e o coeficiente de correlação. As curvas utilizadas na validação foram a de queda de resistência com tempo de ruptura, diagrama de Norton e gráfico de Monkman-Grant, conforme Figuras 5 a 7.

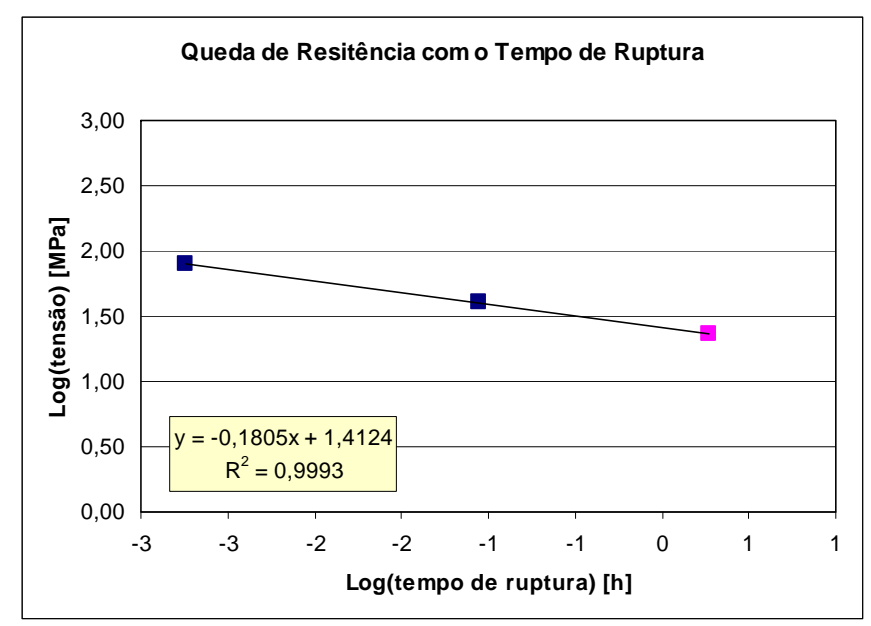

Figura 5: Queda de resistência com o tempo de ruptura na temperatura de $1.100^{\circ} \mathrm{C}$, indicando boa consistência entre os dados de tração a quente (azul) e fluência (rosa). 


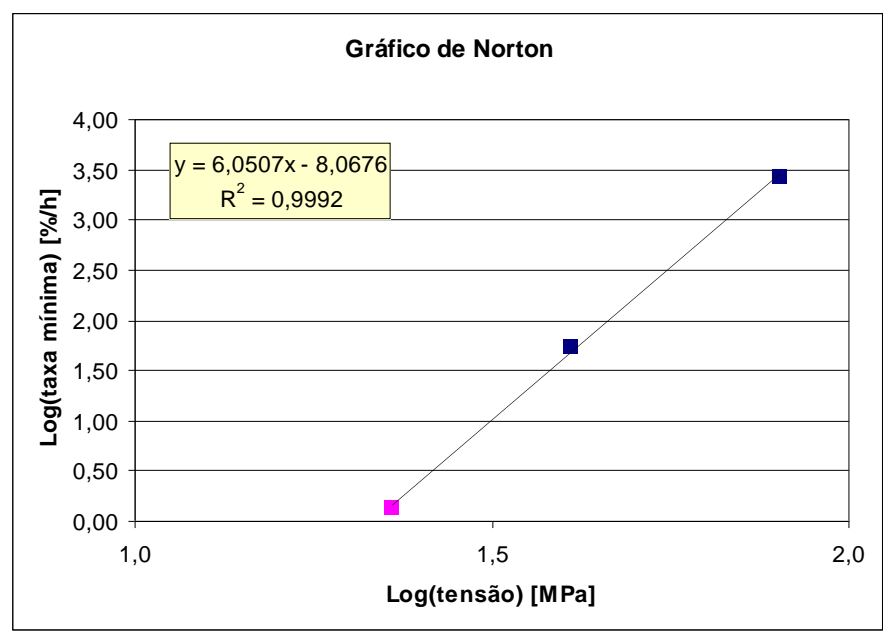

Figura 6: Diagrama de Norton para na temperatura de $1.100^{\circ} \mathrm{C}$. Dados de tração a quente (azul) convertidos e dados de fluência (rosa) mostram boa consistência. Expoente de tensão de 6,0.

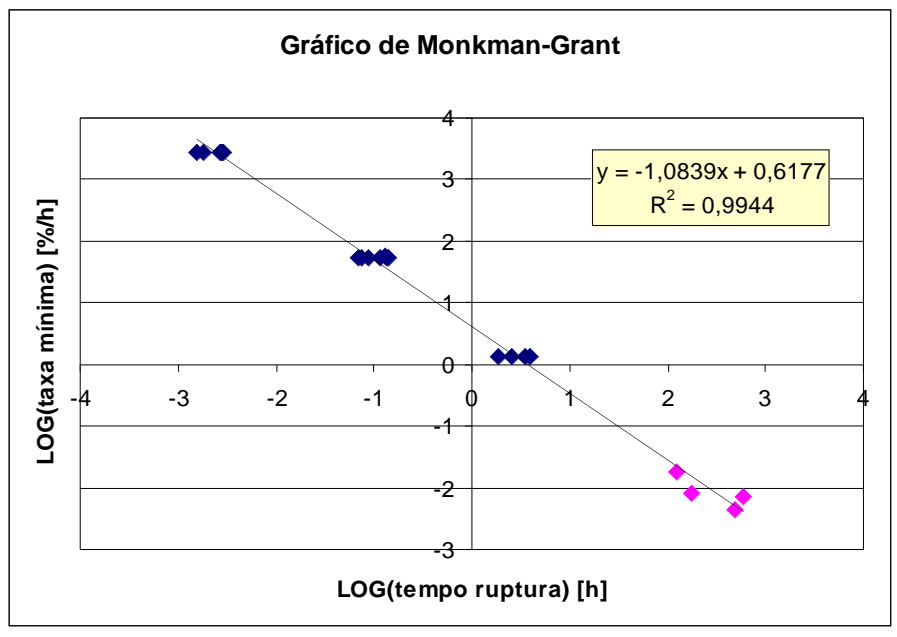

Figura 7: Gráfico de Monkman-Grant correlacionando todos os dados de tração a quente (azul) e fluência (rosa) em todas as condições de ensaio, indicando bom alinhamento com m=1,0839.

Os gráficos de relações clássicas das Figuras 5 a 7 mesclando dados de ensaios de fluência e dados de tração a quente convertidos em fluência mostram boa correlação e semelhança com o comportamento de fluência. A relação de Monkman-Grant, por exemplo, indica que o coeficiente $m$ é igual à unidade para maioria dos metais. Isto também foi evidenciado para este material correlacionando dados de tração a quente em fluência conforme Figura 7.

Uma vez verificado que a correlação de tração a quente e fluência é consistente, é possível construir a curva mestre de Larson-Miller em função da tensão a partir dos dados da Tabela 2 conforme Figura 8. 


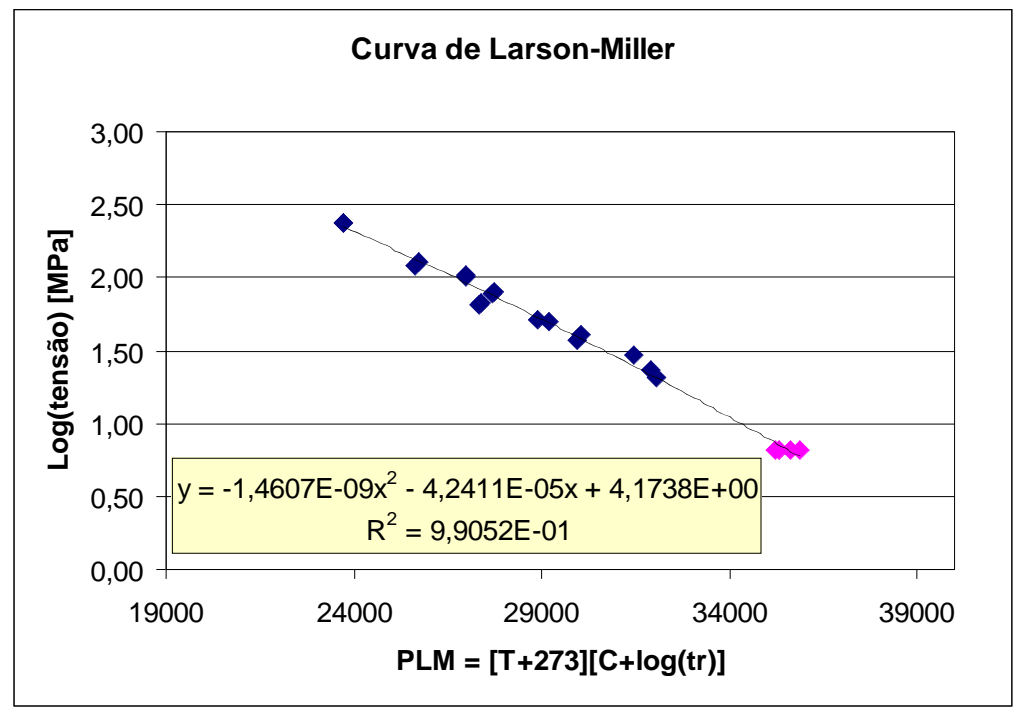

Figura 8: Curva mestre de PLM x Tensão mostrando bom alinhamento entre os dados de tração a quente (azul) e fluência (rosa).

\subsection{Definição da tensão atuante}

Outra variável necessária a avaliação de vida residual é a tensão atuante no tubo. Para defini-la são necessários alguns dados de projeto conforme Tabela 3.

Tabela 3: Dados de projeto dos tubos da radiação do forno reformador.

\begin{tabular}{l|c|c}
\hline PARÂMETRO & VALOR & UNIDADE \\
\hline Pressão de Projeto $(\mathrm{P})$ & 26,3 & $\mathrm{kgf} / \mathrm{cm}^{2}$ \\
\hline Pressão de Operação & 23,0 & $\mathrm{kgf} / \mathrm{cm}^{2}$ \\
\hline Temperatura de Projeto $(\mathrm{T})$ & 972 & ${ }^{\circ} \mathrm{C}$ \\
\hline Diâmetro externo do tubo $(\mathrm{D})$ & 131,6 & $\mathrm{~mm}$ \\
\hline Diâmetro médio do tubo $\left(\mathrm{D}_{\mathrm{m}}\right)$ & 116,0 & $\mathrm{~mm}$ \\
\hline Espessura do tubo $(\mathrm{t})$ & 18,0 & $\mathrm{~mm}$ \\
\hline Limite de Escoamento $\left(\sigma_{\mathrm{y}}\right)$ & $420\left(\mathrm{a} 972^{\circ} \mathrm{C}\right)$ & $\mathrm{kgf} / \mathrm{cm}^{2}$ \\
\hline
\end{tabular}

As tensões foram calculadas utilizando as equações (numerar as equações) conforme item 10.5.2.5a do API-579/ASME FFS-1 (válido para tubos cilíndricos com relação D/t>6):

$$
\begin{aligned}
& \sigma_{1}=\frac{P D_{m}}{2 t} \\
& \sigma_{2}=\frac{\sigma_{1}}{2} \\
& \sigma_{e}=0,866 \sigma_{1}
\end{aligned}
$$

Substituindo-se os valores, obtêm-se: $\sigma_{1}=74,1 \mathrm{kgf} / \mathrm{cm}^{2}, \sigma_{2}=37,1 \mathrm{kgf} / \mathrm{cm}^{2}, \sigma_{\mathrm{e}}=64,0 \mathrm{kgf} / \mathrm{cm}^{2}$. 
Uma vez definidas as tensões atuantes, é necessário verificar se o componente possui proteção adequada contra colapso plástico baseado na tensão de referência. Para tal, a tensão de referência deve ser menor que o limite de escoamento do material na temperatura de análise conforme abaixo:

$$
\sigma_{r e f}^{p}=\frac{P_{b}+\sqrt{P_{b}^{2}+9 P_{L}^{2}}}{3} \leq \sigma_{y s}
$$

Considerando $\mathrm{P}_{\mathrm{L}}=74,1 \mathrm{kgf} / \mathrm{cm}^{2}$ e $\mathrm{P}_{\mathrm{b}}=0,0$, obtém-se que $\sigma_{\text {ref }}^{p}=74,1 \leq \sigma_{y s}$. Portanto, o componente está protegido contra colapso plástico.

\subsection{Avaliação de Vida Residual}

O API-579/ASME FFS-1 Part 10 utiliza o conceito de tensão efetiva a ser utilizada na avaliação de vida residual. Esta tensão é resultado de uma composição das tensões principais com a tensão equivalente de VonMises, e é o valor utilizado para definir o parâmetro de Larson-Miller no gráfico da Figura 8 para extrapolar a vida residual na temperatura desejada.

No entanto, como existem poucos dados sobre o material analisado, principalmente o que se refere à constante C utilizada na equação do parâmetro de Larson-Miller, foi definido conservadoramente que a tensão utilizada na extrapolação do tempo de ruptura será a tensão circunferencial $\left(\sigma_{1}\right)$ de $74,1 \mathrm{kgf} / \mathrm{cm}^{2}$ obtida no item anterior.

Assim, o valor do parâmetro de Larson-Miller obtido da Figura para esta tensão é de 35172. A partir deste valor a vida residual do tubo foi estimada em várias condições de temperatura de operação utilizando a equação:

$$
\log \left(t_{R}\right)=\frac{P L M}{T+273}-C
$$

$\mathrm{T}$ - temperatura de análise em ${ }^{\circ} \mathrm{C}$

$\mathrm{t}_{\mathrm{R}}$ - vida residual em horas

C - constante (22,96 para este material conforme fabricante)

PLM - parâmetro de Larson-Miller (igual a 35172 para tensão de 74,1kgf/cm2)

A Tabela 4 mostra vida residual estimada para alguns valores de temperatura de parede dos tubos:

Tabela 4: Vida residual dos tubos para algumas condições operacionais.

\begin{tabular}{c|c}
\hline $\begin{array}{c}\text { TEMPERATURA } \\
{\left[{ }^{\circ} \mathrm{C}\right]}\end{array}$ & $\begin{array}{c}\text { VIDA RESIDUAL } \\
\text { [ANOS] }\end{array}$ \\
\hline 800 & Infinita \\
\hline 850 & Infinita \\
\hline 900 & Infinita \\
\hline 972 & 22,3 \\
\hline 1000 & 5,3 \\
\hline 1050 & 0,5 \\
\hline 1100 & 0,1 \\
\hline
\end{tabular}

A Tabela acima mostra que para as condições operacionais normais de temperatura de parede dos tubos $\left(800^{\circ} \mathrm{C}\right.$ a $900^{\circ} \mathrm{C}$ durante a campanha) a vida residual é muito longa.

Considerando que o limite de operação é a temperatura de projeto de $972^{\circ} \mathrm{C}$, a vida residual é de 22,3 anos, a partir de março de 2009. 


\section{CONCLUSÕES}

A vida residual dos tubos catalisadores do forno reformador para temperatura de parede no máximo a $972^{\circ} \mathrm{C}$ é estimada em 22,3 anos (3,5 campanhas) e, apesar dos tubos estarem próximos de atingir a vida de projeto (100.000 horas), não há necessidade de substituição imediata.

A avaliação de vida residual utilizando metodologia Omega não é aplicável ao material HP modificado nas condições dos ensaios realizados. Por isso foi utilizada correlação de dados de ensaios de tração a quente e fluência conforme metodologia proposta em [2].

Apesar deste procedimento necessitar de uma avaliação com maior número de dados de ensaios para completa consolidação, mostrou-se uma alternativa prática e viável para realização de uma estimativa de vida residual dos tubos, fornecendo subsídio para decisão de substituição.

\section{AGRADECIMENTOS}

Agradecemos a Petrobras/REPLAN por possibilitar a realização deste trabalho e permitir sua publicação.

\section{BIBLIOGRAFIA}

[1] Part 10: Components Operanting in Creep Range. In: API-579/ASME FFS-1 FITNESS FOR SERVICE, 2ed, 2007.

[2] REIS SOBRINHO, J. F. e BUENO, L., O., Estudo do comportamento de fluência do aço 2,25Cr1Mo.Parte 2: Uma análise baseada em metodologias tradicionais de parametrização. Anais em CDROM: XV CBECIMAT- Congresso Brasileiro de Ciência e Engenharia de Materiais, 09-13 Dezembro 2002, Natal (RN):artigo número 306-021: 1-6.

[3] PRAGER, M., Development of MPC Omega Method for Life Assesment in Creep Range, Journal of Pressure Vessel Technology, vol. 117, p. 95-113, 1995. 